bowel revealed dusky tissue without necrosis. The heart was appreciated through the defect.

The diaphragmatic defect was then primarily closed with figure-of-eight 1-0 silk sutures, ultimately leaving the opposing edges approximated (Figure 2, B). To reinforce the closure, the left triangular ligament of the liver was then fixed to the diaphragm overlying the defect by means of interrupted sutures (Figure 2,C). The patient tolerated the procedure well and was discharged home on postoperative day 4. At 1-month follow-up, the patient was without complications and in normal sinus rhythm.

\section{DISCUSSION}

Hybrid transvenous and surgical myocardial ablation is merely the latest iteration of treating atrial fibrillation. ${ }^{2}$ As with many efficacious novel procedures, the safety profile continues to evolve. The largest published data set, a meta-analysis of 9 studies incorporating 335 patients, found a complication rate of $4.1 \% .^{2}$ The most common complications are minor bleeding and cardiac tamponade, but massive hemorrhage, atrioesophageal fistula, and thermal esophageal injury have also been reported. ${ }^{1}$

As demonstrated, unrepaired diaphragmatic defects pose serious risks after hybrid ablation. No consensus exists, however, regarding optimal closure of intrapericardial diaphragmatic defects. Although small defects can often be closed primarily, ${ }^{3}$ larger defects prove more challenging. Synthetic mesh repair, the criterion standard for large diaphragmatic defect repair, should be avoided in cases of increased risk for infection. ${ }^{3}$ Biologic meshes have been used in such cases ${ }^{3}$ but still have a propensity for complications, including infection, seroma, erosion, and potential explantation. ${ }^{4}$ The use of autologous vascularized tissue performs well in conditions conducive to infection. ${ }^{5}$

The hepatic buttress provides multiple benefits as a diaphragm closure technique without the complications of stand-alone primary or mesh-related repair. Initially, the apertures between sutures can accommodate continuous and passive drainage of effusive fluid, ultimately avoiding life-threatening tamponade. The hepatic ligament reinforces the closure without exposing myocardium or intra-abdominal organs to foreign material, thereby avoiding potential mesh infection, erosion, or dysrhythmia. In addition, the buttress ensures durable closure and greatly reduces the risk of recurrent bowel herniation. The hepatic buttress thus serves as a viable option in treating pericardial diaphragmatic defects, especially after hybrid ablation.

\section{References}

1. Zembala M, Filipiak K, Kowalski O, Boidol J, Sokal A, Lenarczyk R, et al Minimally invasive hybrid ablation procedure for the treatment of persistent atrial fibrillation: one year results. Kardiol Pol. 2012;70:819-28.

2. Gelsomino S, Van Breugel HN, Pison L, Parise O, Crijns HJ, Wellens F, et al. Hybrid thoracoscopic and transvenous catheter ablation of atrial fibrillation. Eur J Cardiothorac Surg. 2014;45:401-7.

3. Al-Nouri O, Hartman B, Freedman R, Thomas C, Esposito T. Diaphragmatic rupture: Is management with biological mesh feasible? Int J Surg Case Rep. 2012;3:349-53

4. Patel KM, Bhanot P. Complications of acellular dermal matrices in abdominal wall reconstruction. Plast Reconstr Surg. 2012;130(5 Suppl 2):216S-24S.

5. Kuo YR, Kuo MH, Lutz BS, Huang YC, Liu YT, Wu SC, et al. One-stage reconstruction of large midline abdominal wall defects using a composite free anterolateral thigh flap with vascularized fascia lata. Ann Surg. 2004;239: $352-8$.

\title{
A case of right atrial compression by the elevated liver successfully treated by diaphragmatic plication
}

\author{
Amr A. Arafat, MD, ${ }^{a}$ Mohamed S. Elkahwagy, MD, ${ }^{a}$ Mohammed H. Sherif, MSc, ${ }^{b}$ and \\ Abdel-Hady M. Taha, MD, ${ }^{\text {a Tanta, Egypt }}$
}

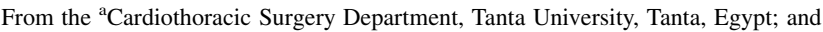
the ${ }^{b}$ Cardiology Department, Tanta University, Tanta, Egypt.

Disclosures: Authors have nothing to disclose with regard to commercial support. Received for publication April 8, 2015; accepted for publication April 18, 2015.

Address for reprints: Amr A. Arafat, MD, Tanta University, Cardiothoracic Surgery Department, Al-Geish St, Tanta, Gharbyia 31529, Egypt (E-mail: amr.arafat@ med.tanta.edu.eg).

J Thorac Cardiovasc Surg 2015;150:e7-10

$0022-5223 / \$ 36.00$

Copyright (C) 2015 by The American Association for Thoracic Surgery

http://dx.doi.org/10.1016/j.jtcvs.2015.04.053
}

Diaphragmatic eventration in old age is a rare anomaly that infrequently requires intervention. The etiology of the eventration could be congenital in origin as a result of failure of development of the muscle of the diaphragm or less frequently secondary to acquired conditions. ${ }^{1,2}$ In most cases diaphragmatic eventration is asymptomatic, with incidental discovery on chest radiography, or it may present with dyspnea, chest infection, gastrointestinal symptoms, ${ }^{2}$ and, rarely, cardiac compression symptoms. ${ }^{3}$ 

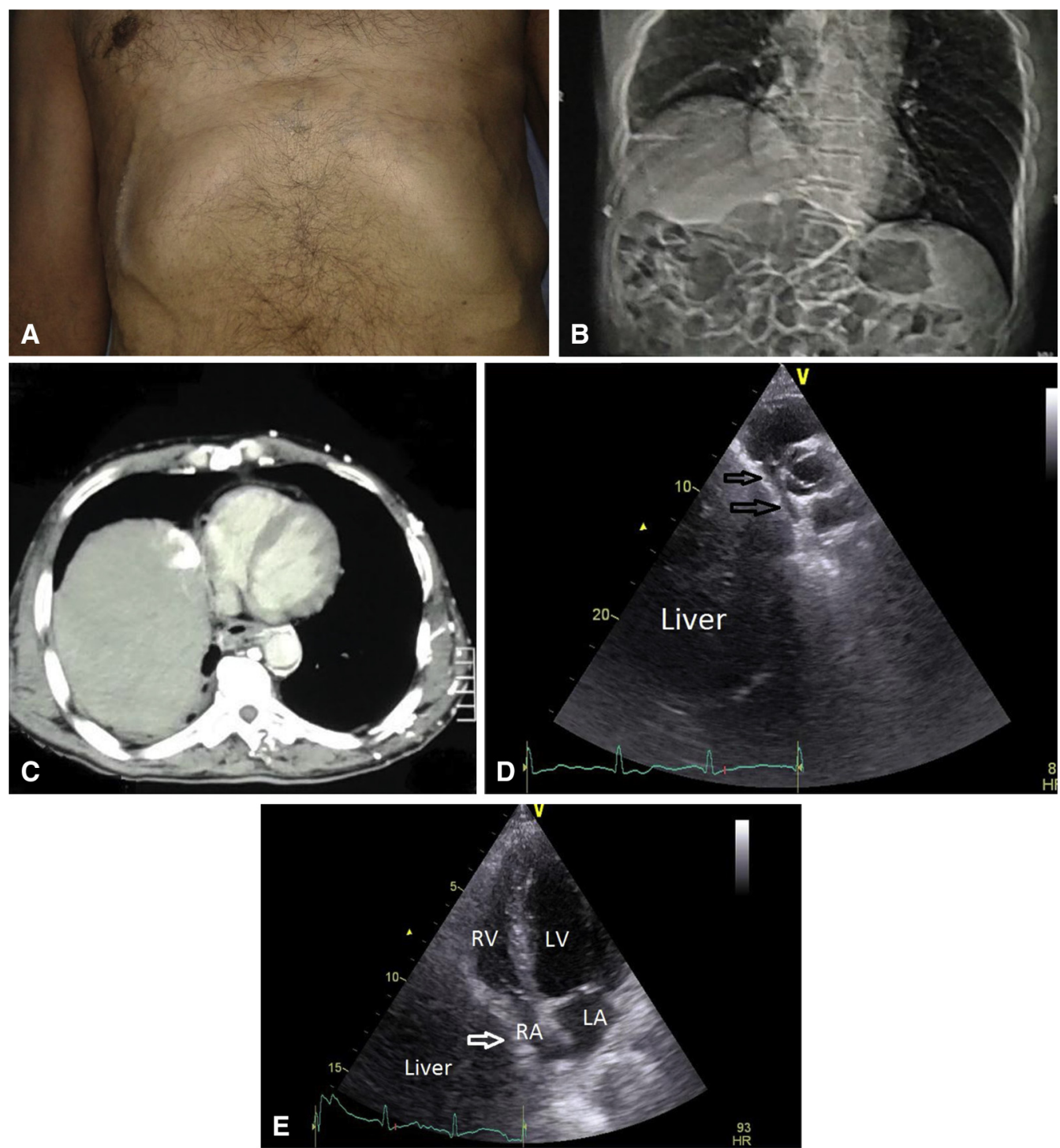

FIGURE 1. Preoperative images. A, Photo of the dilated chest wall veins. B and C, Preoperative computed tomographic scans show elevated diaphragm and dilated veins along the chest wall and internal thoracic arteries, with the liver compressing the right atrium. $D$ and E, Preoperative parasternal short-axis and 4-chamber echocardiographic views show compression of the right atrium by the liver (arrows). $R V$, Right ventricle; $L V$, left ventricle; $R A$, right atrium; $L A$, left atrium.

We report a case of right diaphragmatic eventration in a 64-year-old man with a presentation of obstruction of the orifices of both superior and inferior venae cavae.

\section{CLINICAL SUMMARY}

A 64-year-old man came to our institution with acute dyspnea and right-sided chest pain. The patient was smoker and had a history of inguinal hernia repair 10 years previously with no history of trauma. On clinical examination, the patient had orthopnea and congested veins in the neck and chest wall, with normal cardiac auscultation and decreased breath sounds over the right lower hemithorax. The diagnostic workup revealed a normal 12-lead electrocardiogram and elevated creatine kinase isoenzyme MB and C-reactive protein. Two-dimensional transthoracic echocardiography demonstrated a mass compressing the right atrium and obstructing the inlet of the inferior and superior venae cavae. Computed chest tomographic scan demonstrated an elevated right diaphragmatic copula and the liver compressing the right atrium, with multiple dilated veins on the anterior and lateral chest wall, azygos and hemiazygos veins, and veins along the internal thoracic artery (Figure 1).

We decided to relieve the right atrial compression surgically through an eighth intercostal space thoracotomy incision. Operative inspection demonstrated a thinned right copula of the diaphragm. The right lower lobe was completely atelectatic, and with careful inflation 

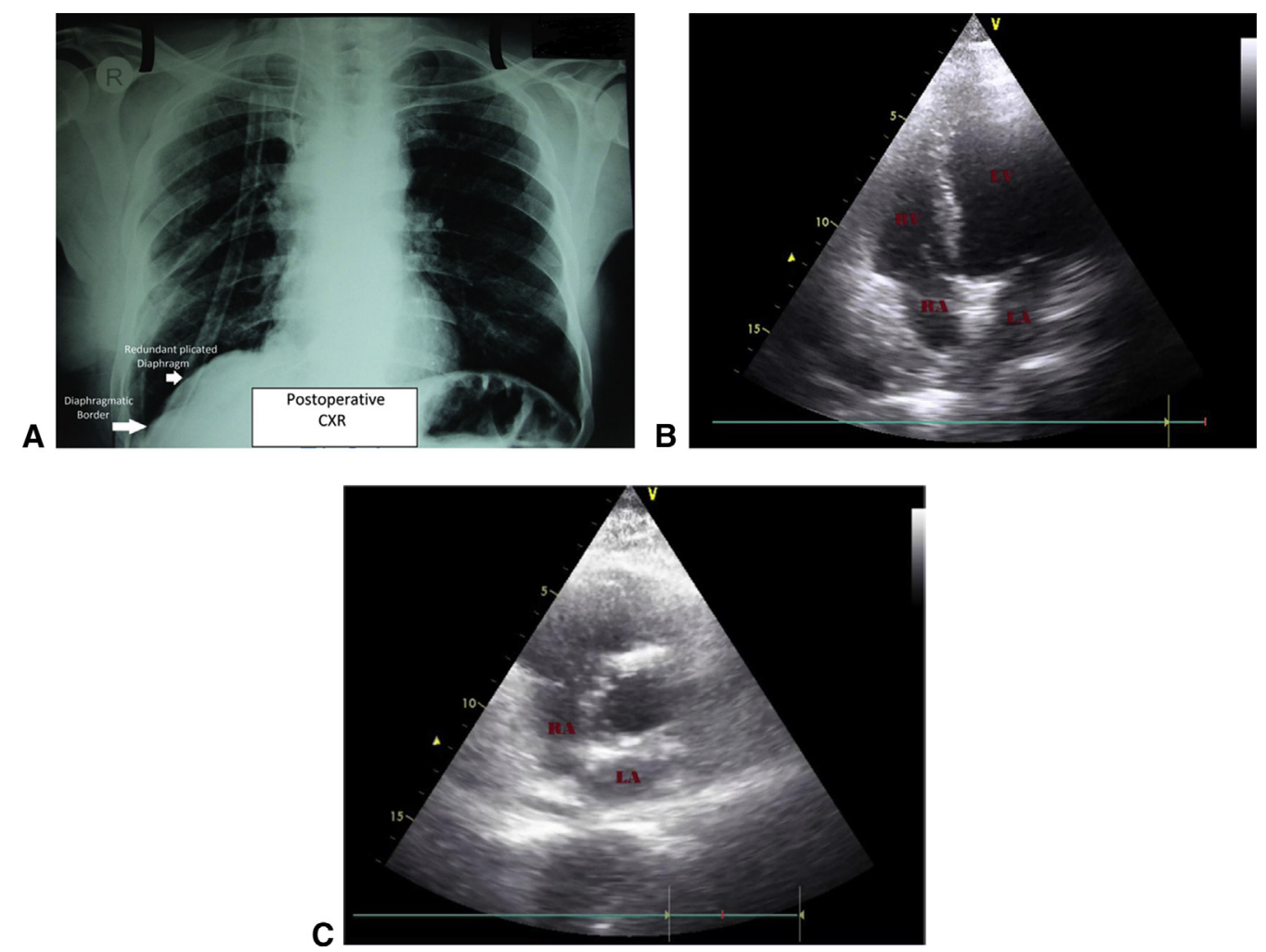

FIGURE 2. Postoperative images. A, Postoperative chest radiograph $(C X R)$ shows marked reduction in the position of the right hemidiaphragm. B and C, Parasternal short-axis and 4-chamber echocardiographic views. $L V$, Left ventricle; $R V$, right ventricle; $R A$, right atrium; $L A$, left atrium.

the lobe expanded fully. Diaphragmatic plication was performed through $5 \mathrm{U}$-shaped anteroposterior sutures. The redundant central diaphragm was then sutured to the lateral diaphragmatic edges, and biopsy of the diaphragm was performed. The patient was extubated intraoperatively and transferred to the postoperative intensive care unit.

The right lower atelectasis had tendency to recur in the first postoperative days, and this was managed with intensive physiotherapy. The patient had uneventful postoperative recovery, with marked improvement of the preoperative symptoms. Postoperative chest radiography demonstrated a reduced right diaphragmatic copula, and the transthoracic echocardiography showed relief of the right atrial compression (Figure 2). Diaphragmatic histologic examination showed fibrotic tissue with scarce, scattered muscle fibers. The patient was discharged on postoperative day 7 and remained symptom free after 6 months of follow-up, with disappearance of the previously congested veins.

\section{DISCUSSION}

Cardiac compression is a rare presentation of diaphragmatic eventration, and it has been reported in a few cases in association with other pathologic processes predisposing toward compression. Right atrial compression has been reported in association with Chilaiditi syndrome ${ }^{3}$ and in a patient with multiple hepatic cystic lesions. ${ }^{4}$ Eventration unusually presents in old age, as in our case, and characteristically the patient didn't have associated lung or abdominal pathology predisposing toward cardiac compression and the elevated liver was normal in size.

The differential diagnoses of the eventration in our patient were pericardial tamponade and myocardial infarction, especially with the elevated creatine kinase isoenzyme $\mathrm{MB}$ and C-reactive protein levels, which could have been caused by muscular injury. Echocardiography, electrocardiography, and chest radiography confirmed the diagnosis in this patient and excluded cardiac pathology. The unique clinical finding in our patient was the dilated cavocaval anastomotic channels on the chest wall, which supports the chronic nature of the condition and draws attention to mechanical obstruction of the systemic venous system.

The exact etiology of diaphragmatic eventration in this case was not clear. The thinned, fibrotic diaphragm found at time of surgical intervention and the histologic examination supported the chronic nature of the condition. On the other hand, the elevated enzymes and the C-reactive protein level at the time of symptom onset supported the presence of acute diaphragmatic muscle insult as the cause of 
the patient's acute symptoms. Most probably, the patient had chronic asymptomatic eventration exacerbated by recent muscle injury, which resulted in the acute venous obstruction.

Diaphragmatic eventration in symptom-free patients does not require surgical intervention. The standard surgical procedure performed is diaphragmatic plication, which is effective in improving pulmonary symptoms and function. ${ }^{2,5}$ Surgical intervention was indicated in our case to relieve the acute right atrial compression. Our case shows that simple diaphragmatic plication is an effective way to relieve the compression and improve the patient's symptoms. Postoperative care is of crucial importance for those patients to prevent lower lobe atelectasis.
In conclusion, right atrial compression with obstruction of the systemic venous drainage is a rare presentation of diaphragmatic eventration, and diaphragmatic plication is effective in relieving the compression.

\section{References}

1. Deslauriers J. Eventration of the diaphragm. Chest Surg Clin N Am. 1998:8:315-30.

2. Calvinho P, Bastos C, Bernardo JE, Eugénio L, Antunes MJ. Diaphragmmatic eventration: long-term follow-up and results of open-chest plication. Eur J Cardiothorac Surg. 2009;36:883-7.

3. Hountis P, Ikonomidis P, Stamatelopoulos A, Douzinas M. Compression of the right atrium due to coexistence of diaphragmatic eventration and Chilaiditi's syndrome. Thorac Cardiovasc Surg. 2008;56:365-7.

4. Nikolić M, Mathewson P, Malhotra A, Belham M. Unusual cause of right heart compression. Cardiol J. 2012;19:441-2.

5. Freeman RK, Wozniak TC, Fitzgerald EB. Functional and physiologic results of video-assisted thoracoscopic diaphragm plication in adult patients with unilateral diaphragm paralysis. Ann Thorac Surg. 2006;81:1853-7; discussion 1857. 